\title{
Effect of Subcutaneous Adrenaline/Saline/Lidocaine Injection on Split-Thickness Skin Graft Donor Site Wound Healing
}

\author{
Kohei Fukuoka,* Shunjiro Yagi,* Yoshiko Suyama,* Wataru Kaida,† Maki Morita* and Ichiro Hisatome \\ *Department of Plastic and Reconstructive Surgery, Tottori University Hospital, Yonago 683-8504, Japan, †Department of Plastic \\ and Reconstructive Surgery, Shimane Prefectural Central Hospital, Izumo 693-0068, Japan, and \$Department of Genetic Medicine \\ and Regenerative Therapeutics, Institute of Regenerative Medicine and Biofunction, Graduate School of Medical Sciences, Tottori \\ University, Yonago 683-8503, Japan
}

\section{ABSTRACT}

Background Subcutaneous injection of tumescent solution, which contains local anesthetic, adrenaline, and saline, before split-thickness skin graft harvesting, shows a significant hemostatic effect. This method can reduce the initial bleeding from the donor site. The aim of this study is to assess the benefits of controlling the bleeding from donor sites by tumescent injection. A randomized, controlled trial was performed to compare the wound healing of split-thickness skin graft donor sites treated with or without tumescent injection.

Methods This randomized, controlled trial examined donor site healing days as the main measure of outcome. postoperative pain, donor site ulceration, and scar quality were evaluated as secondary outcome measures. Patients planned for split-thickness skin graft harvest were randomly assigned to receive either pre-harvest subcutaneous injection of local anesthetic, adrenaline, and saline solution (tumescent solution) (Group 1) or post-harvest application of adrenaline solution-soaked gauze to the skin graft donor sites (Group 2). Donor sites were treated with calcium alginate dressings after graft harvesting. On the 10th postoperative day, the dressings were removed and donor site healing were measured. Follow-up evaluation of scar quality was performed 6 months after surgery. Postoperative pain was evaluated on the 1st day after operating.

Results Forty-five patients (26 males; average age 61.8 years) completed the late follow-up evaluation (6 months postoperatively), with 26 patients in group 1 and 19 in group 2. There were no significant differences between the two groups in any of the outcome measures. Conclusion Tumescent technique provides sufficient hemostasis in split skin graft donor sites, especially the initial bleeding just after graft harvesting, without any negative effects. Larger series should be studied to evaluate the effect in donor site wound healing.

Key words donor site wounds; hemostasis; split-thickness skin grafts; tumescent injection; wound healing
To control the bleeding from split-thickness skin graft donor sites, the use of several hemostatic agents has been reported. ${ }^{1,2}$ Most of these agents are applied to the wound surface after graft harvesting. However, even with these methods, initial bleeding just after graft harvesting cannot be avoided. For hemostasis of splitthickness skin graft donor sites, we prefer subcutaneous adrenaline administration with saline and local anesthetic before graft harvesting (i.e., the tumescent technique). ${ }^{3,4}$ The tumescent technique describes the practice of injecting a dilute solution of adrenaline with local anesthetic subcutaneously until the tissue becomes swollen (tumescent). The hemostasis of this technique is due to both adrenaline-induced vasoconstriction and hydrostatic compression by the tumescent effect. It was originally used for liposuction. Since then, it has been most commonly used for dermatologic surgery for pain relief and hemostasis. Recently, the technique has been applied to a wider range of procedures, such as hair transplantation, ${ }^{5}$ mastectomy, ${ }^{6-8}$ burn excision, ${ }^{9}$ and varicose vein surgery. ${ }^{10}$ The tumescent technique is also used for hemostasis of split-thickness skin graft donor sites. ${ }^{11}$ Compared to topical administration of adrenaline, the use of tumescent solution prior to graft harvest makes an apparent difference in the immediate bleeding just after graft harvesting (Fig. 1). By stopping this initial bleeding from the donor site, contamination of wound dressings can be minimized (Fig. 2). Contaminated wound dressings tend to stick to the wound, and firmly fixed wound dressings could damage the surface during dressing changes. Remaining clots on the wound surface could be a cause of infection. Avoiding these issues would thus help donor site wound healing.

The aim of this study is to evaluate the efficacy of hemostasis by tumescent technique in split-thickness skin graft donor site wound healing. To assess the

Corresponding author: Kohei Fukuoka, MD

kfukuoka@tottori-u.ac.jp

Received 2020 October 14

Accepted 2021 January 6

Online published 2021 February 1 


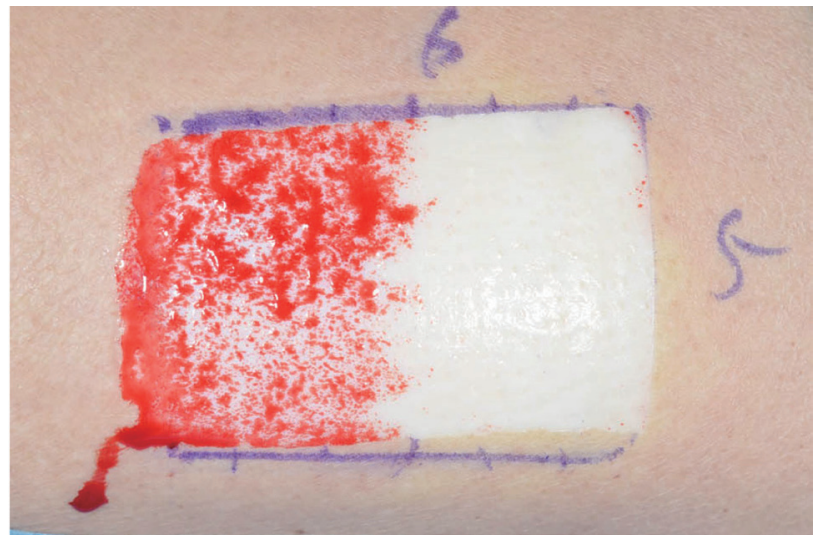

Fig. 1. Difference in bleeding from the split-thickness donor sites. The right side received tumescent injection before graft harvesting, and the left side received only saline injection.

benefits of controlling the bleeding from donor sites, a randomized, controlled trial was performed to compare the wound healing of split-thickness skin graft donor sites treated with or without tumescent injection.

\section{MATERIAL AND METHODS \\ Study design}

This was a prospective, randomized controlled trial. Days required for donor site wound healing was examined as the main measure for outcome. Postoperative pain, donor site ulceration, and scar quality were evaluated as the secondary outcome measures.

\section{Patients selection criteria}

For this study, patients admitted to Tottori University Hospital and Shimane Prefectural Central Hospital between April 2013 and March 2016 with skin defects and planned for split-thickness skin graft harvest from the thigh were recruited.

The study was approved by the Institutional Review Board at Tottori University Hospital (approval number 2399).

Patients who met the following selection criteria were included: no skin disease or skin trouble that could affect wound healing; informed consent could be obtained; age between 20 and 84 years; and donor site size $\geq 20 \mathrm{~cm}^{2}$. Individuals who refused to participate in the study and participants who were judged not suitable for the study were excluded. When the experiment or postoperative follow-up was unable to be continued due to interruptions such as the participant's death or a hospital transfer, such cases were also excluded from the study.

Before the operative procedure, informed consent was obtained from the study participants using a

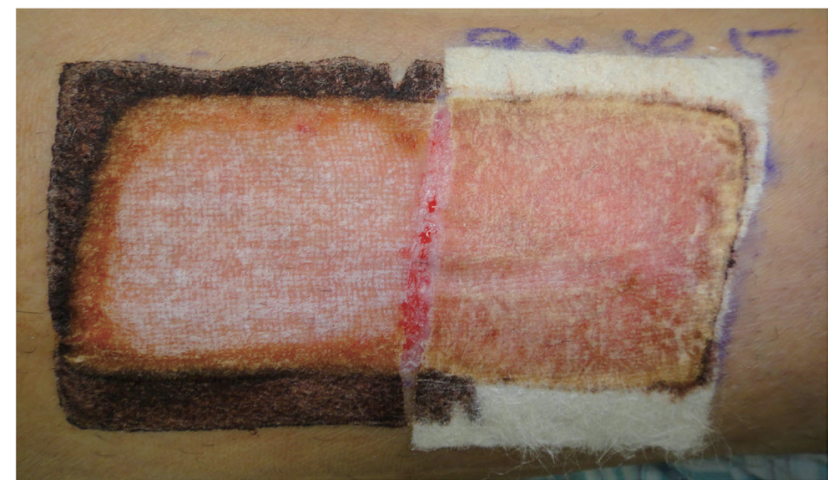

Fig. 2. Differences in wound dressings. Dressings on the donor site with tumescent solution (right side) have minimal contamination. Dressings on the donor site without tumescent injection tend to be firm and stuck to the wound surface.

document showing the details of the treatment and the research. After participants' consent was obtained, registration numbers were given to each participant. They were then randomly assigned to receive either pre-harvest subcutaneous injection of local anesthetic, adrenaline, and saline solution (tumescent solution) (Group 1) or post-harvest application of adrenaline solution-soaked gauze to the skin graft donor sites as a hemostatic procedure (Group 2).

\section{Operation procedure}

The grafting procedure was performed under general anesthesia according to the standard procedure in our institution. In Group 1, tumescent solution $(0.9 \%$ injectable saline with $0.05 \mathrm{~mL}$ adrenaline 1:1000, $50 \mathrm{~mL} \mathrm{1 \%}$ lidocaine in $1000 \mathrm{~mL}$ ) was injected subcutaneously before skin graft harvesting. In Group 2, saline solution ( $0.9 \%$ injectable saline) was injected subcutaneously before harvesting, and epinephrine solution-soaked gauze $(0.9 \%$ injectable saline with $10 \mathrm{~mL}$ adrenaline $1: 1000$ in $1000 \mathrm{~mL}$ ) was topically applied to the wound after graft harvesting. The skin graft was harvested with an electric dermatome (Zimmer Surgical, Inc., Dover, $\mathrm{OH}$ ) at a thickness of 12/1000ths of an inch. After the graft was taken, a calcium alginate dressing (AlgoDERM, Smith \& Nephew, London, UK) was applied to the donor sites. The donor sites were then dressed in sterile gauze and fixed with medical bandages (Fig. 3).

\section{Evaluation methods}

On the 10th postoperative day, the dressing was opened to check the wounds. Photographs of the donor site were then taken every day to assess donor site healing. Wounds were judged as healed when the remaining ulcer area was under $1 \mathrm{~cm}^{2}$. Evaluation of scar quality 


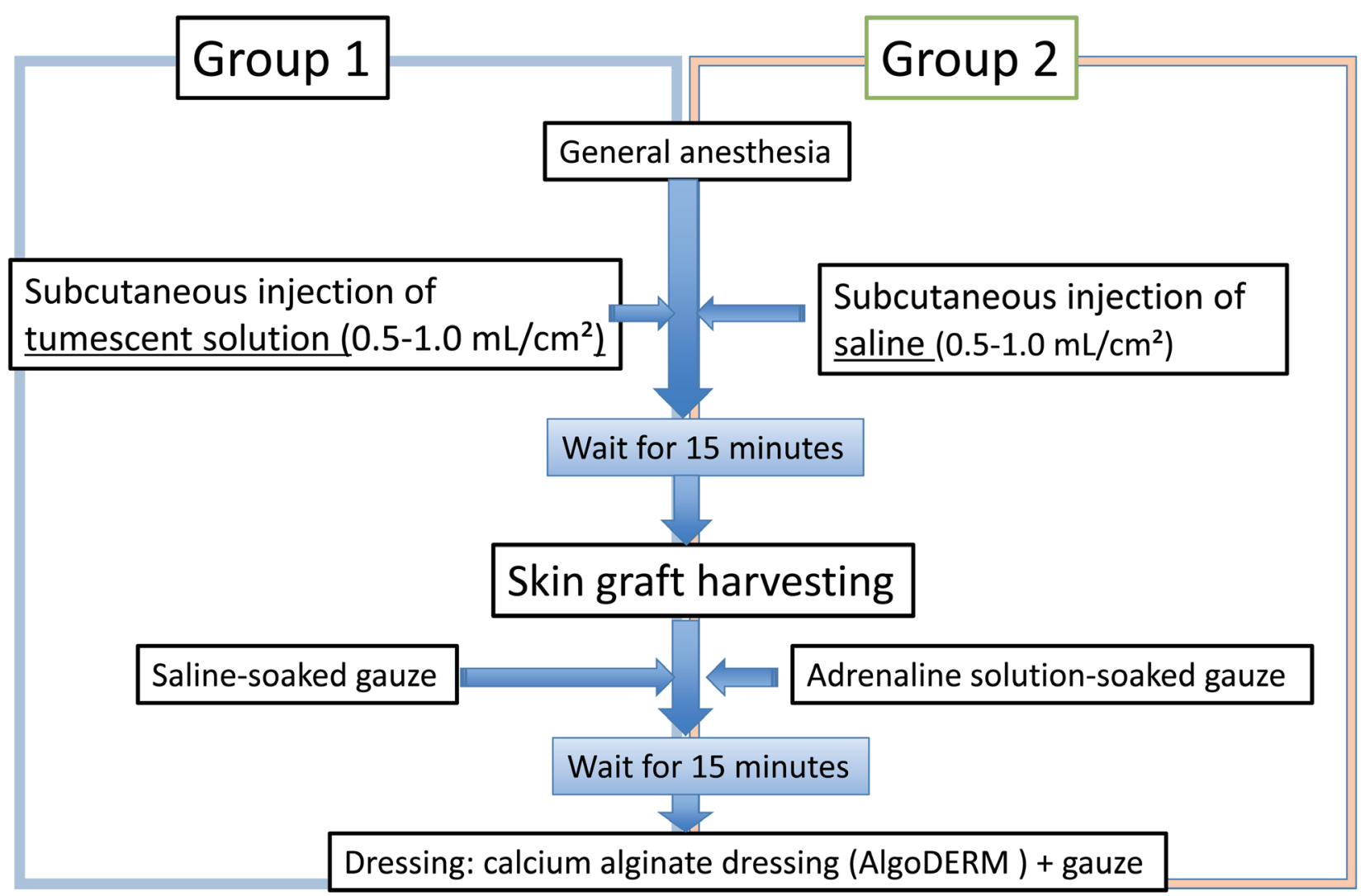

Fig. 3. Operative procedure. Group 1 received tumescent solution injection subcutaneously before skin graft harvesting. Group 2 received saline solution injection subcutaneously before harvesting, and adrenaline (epinephrine) solution-soaked gauze was applied after graft harvesting. Skin grafts were harvested with a Zimmer electric dermatome at a thickness of 12/1000th of an inch. Calcium alginate dressing was applied to the donor site 15 minutes after graft harvesting in both groups.

(hypertrophy and/or ulceration formation, wound photograph data) was performed at least 6 months after surgery. The evaluators were selected from among the nurses in the plastic surgery department. Postoperative pain was assessed subjectively on the $1^{\text {st }}$ postoperative day, using a 10 -grade linear scale $(0=$ little or no pain, $10=$ intolerable pain); the data were obtained from a nurse interview form. Data analysis was performed using statistical analysis software (Stat Flex ver. 6, Artech Co., Osaka, Japan). Mann-Whitney $U$ test or Fisher's exact test for paired samples was used to determine whether there were any differences between the two groups. P values of $<0.05$ were considered significant.

\section{RESULTS}

\section{Patients characteristics}

A total of 49 patients (33 male and 16 females; average age 63.3 years) from Tottori University Hospital and Shimane Prefectural Central Hospital with various skin defects were included in the study. Of them, 45 patients (26 male and 19 females; average age 61.8 years) completed the late follow-up evaluation (6 months after operation). Four patients could not complete the followup evaluation; 1 patient died during the follow-up period, and 3 patients did not come to the outpatient clinic after being discharged or transferred (Fig. 4). Group 1 consisted of 26 patients, and Group 2 consisted of 19 patients. There were no significant differences between the two groups in age, sex, wound size. The most frequent cause of skin defect was burns in both groups (Table 1).

\section{Analysis of the outcome measures}

Donor sites healed within an average of 14.8 days in group 1 and 20.4 days in group 2, with no significant difference between the two groups. Average postoperative pain reported on day 1 was 3.46/10 in group 1 and 2.84/10 in Group 2, with no significant difference.

In the late follow-up period, ulceration of the healed site occurred in 9 cases in both groups (Group $134.6 \%$, Group 2 47.4\%). Hypertrophic scar formation was seen in one patient in Group 2. There was no significant difference between the two groups (Table 


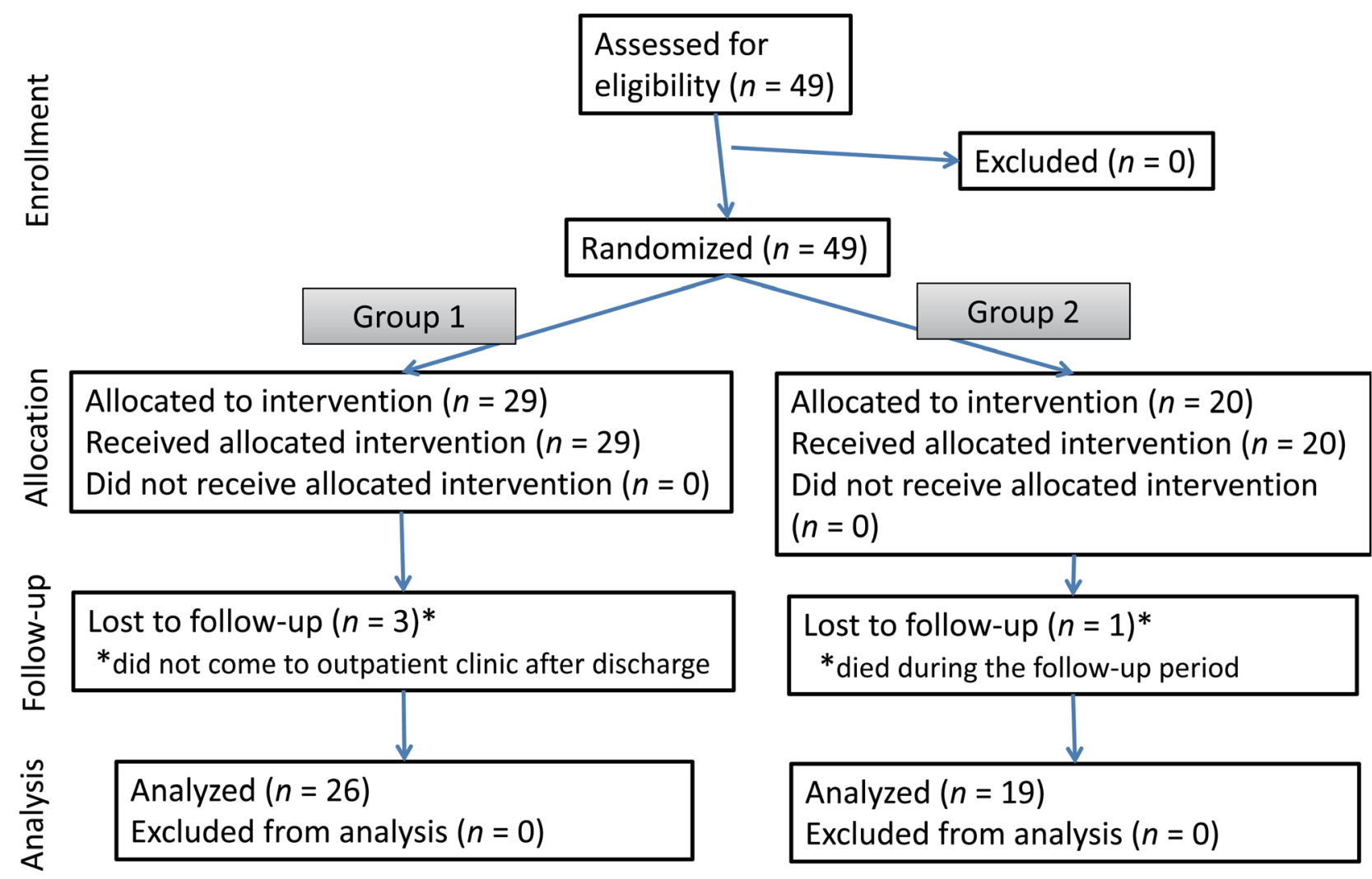

Fig. 4. Participant flow chart according to the CONSORT (Consolidated Standards for Reporting Trials) guidelines. ${ }^{12}$ The number of patients who completed the follow-up period is 26 in group 1 and 19 in group 2.

Table 1. Comparison between participants randomized to group 1 (tumescent injection to donor sites) or group 2 (topical administration of adrenaline)

\begin{tabular}{llll}
\hline & Group 1 $(n=26)$ & Group 2 $(n=19)$ & $P$-value \\
\hline Age $(\mathrm{y})$ & $59.2(20-84)$ & $64.5(38-84)$ & 0.294 \\
Sex (male: female) & $18: 8$ & $13: 6$ & 1.000 \\
Wound size $\left(\mathrm{cm}^{2}\right)$ & $139.1(35-250)$ & $101(24-200)$ & 0.692 \\
Cause of skin defect $(n)$ & Burn 20 (77\%) & Burn 16 (84\%) & \\
& Trauma 2 (7\%) & Foot ulcer 2 (11\%) & \\
& Foot ulcer 1 (4\%) & Necrotizing fasciltis 1 (5\%) & \\
& Malignancy 1 (4\%) & & \\
& Necrotizing fasciltis 1 (4\%) & & \\
& Cronic pyoderma 1 (4\%) & & \\
\hline
\end{tabular}

y, years.

2). One infection occurred in Group 2. No cardiac arrhythmias or significant increases in heart rate were observed following the injection of adrenaline. No other side effects were reported.

\section{DISCUSSION}

This present study compared wound healing in two different hemostatic techniques: the tumescent technique and topical application of adrenaline solution. Both methods have sufficient hemostatic effect, but tumescent injection can control the initial bleeding just after graft harvesting. Compared to topical administration of adrenaline, the use of tumescent solution prior to graft harvest makes an apparent difference in the immediate bleeding just after graft harvesting. We expected improvement in donor site wound healing by controlling 
Table 2. Comparison of the outcome measures and side effects between group 1 and group 2

\begin{tabular}{lllll}
\hline & & Group 1 $(n=26)$ & Group 2 $(n=19)$ & $P$-value \\
\hline $\begin{array}{l}\text { Outcome } \\
\text { measures }\end{array}$ & Healing time (days) & 14.8 & 20.4 & 0.15 \\
& Postoperative pain & 3.46 & 2.84 & 0.33 \\
& Ulceration $(n)$ & $9(34.6 \%)$ & $9(47.4 \%)$ & 0.68 \\
& Hypertrophic scar $(n)$ & $0(0 \%)$ & $1(5.3 \%)$ & 0.42 \\
\hline $\begin{array}{l}\text { Negative } \\
\text { effects }\end{array}$ & Infection $(n)$ & $0(0 \%)$ & $1(5.3 \%)$ & 0.42 \\
\hline
\end{tabular}

this initial bleeding, but the results of this present study showed no significant difference in donor site wound healing or scar quality. Possible reasons of this result are the small sample size, and the use of hemostatic procedure for both groups.

There are several reports that suggest the effectiveness of adrenaline injection before skin graft harvesting. Groenewold et al. performed a systematic review to assess the effectiveness of hemostatic agents for splitthickness skin graft donor sites. They reported that adrenaline and fibrin sealant showed more effective hemostasis than other agents. ${ }^{2}$ Gacto et al. reported that the re-epithelialization of the split-thickness skin graft donor site was faster in a group treated with adrenaline before graft harvesting than in a group treated with saline. ${ }^{13}$ The group that received subcutaneous adrenaline injection needed fewer dressing changes than the control group and thus required shorter time for epithelialization.

In the present study, there was neither delayed healing nor any negative effect on scar quality in those treated with subcutaneous injection of tumescent solution. It is known that moderate bleeding is a necessary process for wound healing. ${ }^{14}$ Platelets secrete several growth factors, such as vascular endothelial growth factor (VEGF), platelet-derived growth factor (PDGF), and transforming growth factor- $\beta$ (TGF- $\beta$ ). These factors promote neovascularization, cell proliferation, and chemotaxis. ${ }^{15}$ Hemostasis by tumescent technique might impede these functions, and may therefore result in delayed wound healing of the donor sites. In response to this concern, Blome-Eberwein et al. examined the effects of adrenaline/saline/local anesthetic solution on donor site perfusion and wound healing. ${ }^{11}$ They showed that subcutaneously injected adrenaline/saline/local anesthetic solution did not affect donor site healing time or long-term scar quality.

Due to the effect of local anesthetics in the tumescent solution, postoperative pain relief was expected. However, there was no significant effect. As possible reasons for this result, the reaction time and the types of anesthetics may be considered. In addition, pain evaluation limited to the skin graft donor site was difficult, because every case had other wounds that were larger than the skin graft donor sites.

As a supplemental effect of tumescent technique, subcutaneous infiltration of tumescent solution can facilitate dermatome contact by raising contoured areas. This procedure thus simplifies skin harvest from the abdomen or back and allows the surgeon to take thinner grafts.

There are several limitations in this study. First, the number of cases was too small. Required sample size was 70 cases in each group according to the sample size calculation program. ${ }^{16}$ It was not able to achieve the required sample size within the study duration. Small sample size possibly affected the result of the analysis. Second, both groups received hemostatic agents, and application of wound dressings with hemostatic effect. To investigate the advantage of the tumescent technique, we planned to nominate the non-hemostatic procedure group as the control. But the plan was rejected by the Institutional Review Board from an ethical perspective. Calcium alginate dressings contain a blood clotting factor; this selection of wound dressing made the difference insignificant between the two groups.

In conclusion, injecting tumescent solution before graft harvesting provides guaranteed hemostasis, including the initial bleeding just after graft harvesting, without any negative effects. This method can be used routinely for split-thickness skin graft harvesting. A larger population of patients should be studied to evaluate the effect of tumescent technique in wound healing.

The authors declare no conflict of interest.

\section{REFERENCES}

1 Netscher DT, Carlyle T, Thornby J, Bowen D, Harris S, Clamon J. Hemostasis at skin graft donor sites: evaluation of topical agents. Ann Plast Surg. 1996;36:7-10. DOI: 10.1097/00000637-199601000-00002, PMID: 8722976 
2 Groenewold MD, Gribnau AJ, Ubbink DT. Topical haemostatic agents for skin wounds: a systematic review. BMC Surg. 2011;11:15. DOI: 10.1186/1471-2482-11-15, PMID: 21745412

3 Klein JA. The tumescent technique. Anesthesia and modified liposuction technique. Dermatol Clin. 1990;8:425-37. DOI: 10.1016/S0733-8635(18)30474-1, PMID: 2199105

4 Conroy PH, O’Rourke J. Tumescent anaesthesia. Surgeon. 2013;11:210-21. DOI: 10.1016/j.surge.2012.12.009, PMID: 23375489

5 Khanna MD. Hair transplantation surgery. Indian J Plast Surg. 2008;41(suppl):56-63. DOI: 10.1055/s-0039-1700476, PMID: 20174544

6 Carlson GW. Total mastectomy under local anesthesia: the tumescent technique. Breast J. 2005;11:100-2. DOI: 10.1111/ j.1075-122X.2005.21536.x, PMID: 15730454

7 Khavanin N, Fine NA, Bethke KP, Mlodinow AS, Khan SA, Jeruss JS, et al. Tumescent technique does not increase the risk of complication following mastectomy with immediate reconstruction. Ann Surg Oncol. 2014;21:384-8. DOI: 10.1245/s10434-013-3311-0, PMID: 24121881

8 Vargas CR, Koolen PGL, Ho OA, Ricci JA, Tobias AM, Lin SJ, et al. Tumescent mastectomy technique in autologous breast reconstruction. J Surg Res. 2015;198:525-9. DOI: 10.1016/j.jss.2015.03.050, PMID: 25891675

9 Robertson RD, Bond P, Wallace B, Shewmake K, Cone J. The tumescent technique to significantly reduce blood loss during burn surgery. Burns. 2001;27:835-8. DOI: 10.1016/ S0305-4179(01)00057-2, PMID: 11718986
10 Roth SM. Endovenous radiofrequency ablation of superficial and perforator veins. Surg Clin North Am. 2007;87:1267-84, xii. DOI: 10.1016/j.suc.2007.07.009, PMID: 17936486

11 Blome-Eberwein S, Abboud M, Lozano DD, Sharma R, Eid S, Gogal C. Effect of subcutaneous epinephrine/saline/local anesthetic versus saline-only injection on split-thickness skin graft donor site perfusion, healing, and pain. J Burn Care Res. 2013;34:e80-6. DOI: 10.1097/BCR.0b013e31825d5414, PMID: 23237817

12 Moher D, Schulz KF, Altman DG. The CONSORT statement: revised recommendations for improving the quality of reports of parallel-group randomised trials. Lancet. 2001;357:1191-4. DOI: 10.1016/S0140-6736(00)04337-3, PMID: 11323066

13 Gacto P, Miralles F, Pereyra JJ, Perez A, Martínez E. Haemostatic effects of adrenaline-lidocaine subcutaneous infiltration at donor sites. Burns. 2009;35:343-7. DOI: 10.1016/ j.burns.2008.06.019, PMID: 18950945

14 Sorg H, Tilkorn DJ, Hager S, Hauser J, Mirastschijski U. Skin wound healing: an update on the current knowledge and concepts. Eur Surg Res. 2017;58:81-94. DOI: 10.1159/000454919, PMID: 27974711

15 Pierce GF, Mustoe TA, Altrock BW, Deuel TF, Thomason A. Role of platelet-derived growth factor in wound healing. J Cell Biochem. 1991;45:319-26. DOI: 10.1002/jcb.240450403, PMID: 2045423

16 Dupont WD, Plummer WD Jr. Power and sample size calculations. Control Clin Trials. 1990;11:116-28. DOI: 10.1016/01972456(90)90005-M, PMID: 2161310 